\title{
Letter
}

\section{Shouldn't we be using more capillary electrophoresis nowadays?}
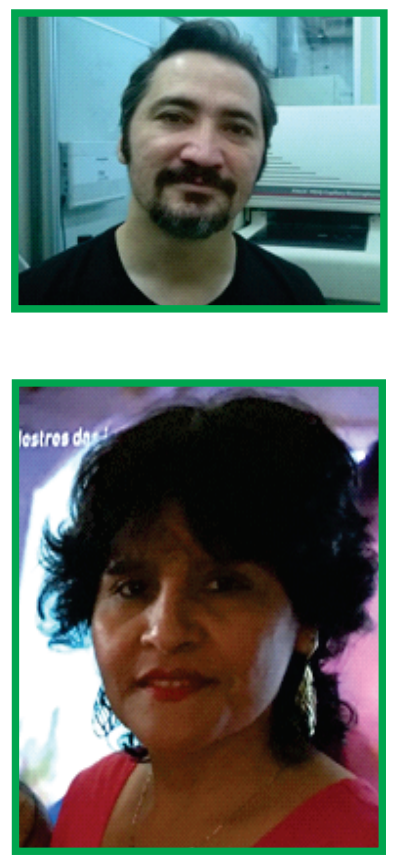

José Roberto Rodrigues de Souza

School of Pharmaceutical Science

University of São Paulo (USP), SP, Brazil

roberto_souza@usp.br

\author{
Maria Segunda Aurora Prado \\ Professor at the School of Pharmaceutical Science \\ University of São Paulo (USP), SP, Brazil \\ msaprad06@usp.br
}

Capillary Electrophoresis (CE) has been used for different and specific applications presenting also some specific advantages, but it is still an underestimated technique in the analytical fields. We have explored recent applications and advantages of CE use in Science and some comparisons to other techniques.

The search for greener methods and technologies, which could avoid unnecessary use of chemicals and toxic substances, is an important and current issue in Science. Capillary Electrophoresis (CE) is nowadays still an underestimated technique in the analytical field. Many papers have described the advantages of the technique in comparison with high performance liquid chromatography (HPLC) for some cases. Some of those could be described as composing of a relatively simple equipment, cheap capillary columns, fast separations, wide spectrum of analytes types that can be analyzed, simple sample preparation and maybe one of its best advantages is that it is a much cleaner method compared to HPLC $[1,2]$. Regarding the use of organic solvents, CE will require lower amounts compared to HPLC, and many analyses can be conducted only in aqueous media. CE can also be coupled to many detection formats such as MS detection, UV absorbance, electrochemical and others, what makes it so useful as HPLC, sometimes for the same applications.

HPLC is undoubtedly a powerful technique that has multiple applications but CE has gained more attention in the last years due to its advantages and for specific applications. We have listed some of its recent applications in different analytical fields (Table I).

Wang et al. [2] have reviewed CE applications for vitamins analysis. They observed that Microemulsion electrokinetic chromatography (MEEKC) or Capillary electrochromatography (CEC) were the best CE techniques to analyze fat-soluble vitamins and could be used in the simultaneous determination of watersoluble and fat-soluble vitamins even though vitamins analysis by CE showed to be limited in its sensitivity. 
Table I - Recent applications in Capillary Electrophoresis

Hemoglobinopathy screening

Vitamin analysis

Serum N-glycans identification

Clinicalmetabolomics

Enantioseparation

Serum proteins analysis
You-Qiong et al., 2016

Wang et al., 2018

Snyder et al., 2017

Ramautar, 2016

Zhang, 2018

Regenite \& Siede, 2018

Farcaş et al., 2017

CE has also been used for serum analyses [1,3]. You-Qiong et al. [1] conducted a study to compare detection and quantification of hemoglobin New York by CE and HPLC. All 15 cases (100\%) were detected by CE, whereas none was detected by HPLC so, they concluded that CE may be the preferred method for hemoglobinopathy screening in areas with high prevalence of $\mathrm{Hb}$ New York. Another important application of CE coupled with mass spectrometry is its use in clinical metabolomics. Ramauter [4] reviewed its use to screen metabolites in biological samples.

Enantioseparation has also been studied by CE. Zhang et al. [5] have analyzed the use of ionic liquids (ILs) for CE separation in different modes. They reported that chiral ILs was superior than achiral ones because chiral ILs can usually bring extra enantio recognition capability to the separation.

Regeniter \& Siede [6] reviewed CE applications for serum protein analysis and they highlighted some CE advantages over agarose electrophoresis. They reviewed the basic concepts to correctly identify irregularities, monoclonal and oligoclonal peaks. According to them, detection of monoclonal components has been largely improved by capillary electrophoresis and it estimates monoclonal peaks more accurately. CE also provided higher resolution and increases sensitivity for the recognition of monoclonal proteins.

Drug discovery theme has also been studied with CE applications. Farcaş et al. [7] have recently overviewed the use of CE in this context. They confirmed the low use of CE in this field though it has been seen as a very efficient and resolutive separation technique. Reasons for that were reported to be the relatively low number of experienced CE practitioners, the maturity of HPLC in the pharmaceutical industry and some intrinsic limitations of the technique. They reviewed the use of CE for bioassays, drug-plasma interactions and drug metabolism studies. Some interesting CE applications in this field would be to use a portion of its capillary as a micro-reactor, the study of protein-protein interactions and its use to determine the affinity of a tested drug for the targeted protein. Other CE advantages include high throughput, high speed and low quantities of samples and reagents requirements, making CE a cost-effective technique. Additionally, CE enables the study of non-covalent interactions directly in solution under near physiological conditions.

As have been reported in this note, CE represents an efficient, clean and cost-effective technique for many applications. More attention should be paid by the researchers to the use of this technique in their studies and the use in the pharmaceutical industry should be promoted. One of its more important advantages to be pointed is the much lower use of organic solvents and this is becoming a big issue in many research labs. In that way, the search for greener methods is of urgent importance and the increase in the use of CE analytical methods can offer its contribution. 


\section{Letter}

\section{REFERENCES}

1. You-Qiong, L.; Hui-Ping, H.; Zhi-Zhong, C. Clin. Chem. Lab. Med., 2016, 54 (1), pp 91-95.

2. Wang, X.; Li, K.; Yao, L.; Wang, C.; Van Schepdael, A. J. Pharm. Biomed. Anal., 2018, 147, pp 278-287.

3. Snyder, C. M.; Zhou, X.; Karty, J. A.; Fonslow,B. R.; Novotny, M. V.; Jacobson, S. C. J. Chromatog. A, 2017, 1523, pp 127-139.

4. Ramautar, R. Capillary Electrophoresis-Mass Spectrometry for Clinical Metabolomics. In: Makowski, G. S. (Ed.). Advances in Clinical Chemistry, v. 74, Elsevier, 2016, pp 1-34.

5. Zhang, Q.TrAC, Trends Anal. Chem., 2018, 100, pp 145-154.

6. Regeniter, A.; Siede, W.H. Clin. Biochem., 2018, 51, pp 48-55.

7. Farcaş, E.; Pochet, L.; Crommen, J.; Servais, AC.; Fillet, M. J. Pharm. Biomed. Anal, 2017, 144, pp 195-212. 